\title{
Campaña de comunicación para la elección de una carrera técnica de pregrado en la Universidad Politécnica Salesiana
}

\section{Communication campaign for the selecting of a technical undergraduate degree at the Salesian Polytechnic University}

Propuesta

\author{
Adriana Priscila Vallejo \\ Universidad Politécnica Salesiana - Ecuador \\ avallejo@ups.edu.ec
}

Recibido: 16 de octubre de 2015 / Aceptado: 29 de junio de 2016

\section{Resumen}

El presente trabajo tiene como objeto poner a disposición de la comunidad científica, una campaña de comunicación diseñada en base a los factores que intervienen en la elección de una carrera técnica de pregrado en la Universidad Politécnica Salesiana Sede Cuenca. Dado el impulso que actualmente brinda el gobierno ecuatoriano a los Institutos técnicos y tecnológicos del país, es preciso tomar medidas que incentiven la preparación académica de los estudiantes optando no únicamante por una formación técnica sino por una ingeniería lo que asegura profesionales que garanticen los derechos estipulados en la Constitución Política del Ecuador y ayuden a dinamizar la economía con innovación, investigación y emprendimiento.

La investigación es de tipo cualitativo, se apoya en grupos focales y entrevistas semi-estructuradas; su base conceptual está definida por los enfoques de marketing de servicios y relaciones públicas, lo que supone que los factores antes mencionados constituyen el principal elemento para el desarrollo de una propuesta de comunicación creativa que aporte mayor valor a la oferta de la Institución.

Palabras clave: Carreras técnicas, marketing de servicios, relaciones públicas, grupos de interés.

\footnotetext{
Abstract

This paper aims to make available to the scientific community, a communication campaign designed based on the factors involved in choosing a technical undergraduate degree at the Salesian Polytechnic University in Cuenca. Given the momentum that currently provided by the Ecuadorean government to technical and technological country institutes, must take measures to encourage the academic preparation of students opting not only for technical training but by engineering which ensures professionals that guarantee the rights stipulated in the Constitution of Ecuador and help boost the economy with innovation, research and entrepreneurship.

The research is qualitative. It is based on focus groups and semi-structured interviews; its conceptual basis
} 
is defined by marketing approaches and public relations services, which means that the above factors constitute the main element for the development of a creative communi- cational approach to provide a more valuable study fields of the institution.

Keywords: Technical carreers, marketing services, public relations, stakeholders.

Forma sugerida de citar: Vallejo, Adriana (2016). Campaña de comunicación para la elección de una carrera técnica de pregrado en la Universidad Politécnica Salesiana. Alteridad, 11(1), pp. 110-120.

\section{Introducción}

En la actualidad el Ecuador ha colocado como uno de sus principales objetivos el cambio de la matriz productiva, históricamente ha seguido un modelo económico sostenido por la exportación de productos primarios e importación de otros transformados que no han permitido un desarrollo igualitario a escala mundial, así también el nuevo enfoque propuesto dentro del ámbito de la educación, el proyecto Emblemático de Reconversión de la Educación Técnica y Tecnológica Superior Pública del Ecuador apuntan al cumplimiento del Plan Nacional para el Buen Vivir (Secretaría de Educación Superior, 2014) (Samaniego, 2013).

Hoy en día, la industria ecuatoriana tiende al crecimiento, varias empresas están en este proceso $\mathrm{y}$ otras tienen mayor incidencia positiva en el PIB Industrial como las de fabricación de maquinaria, equipo y material de transporte (Banco Central del Ecuador, 2015). Como consecuencia de este crecimiento, también se ha visto beneficiada la demanda de mano de obra técnica, lo que favorece a la Universidad Politécnica Salesiana (UPS) al contar con la oferta académica indicada, de ahí, que se define como pregunta de investigación ¿cuáles son los factores que intervienen en la elección de una carrera de este tipo? Los resultados facilitan la estructura de una propuesta que estimule en positivo la decisión del público objetivo.

En atención a lo antes mencionado, se desarrolló una investigación para identificar los factores que intervienen en la elección de una carrera técnica de pregrado en la Universidad Politécnica Salesiana Sede Cuenca; a partir de lo cual se genera una propuesta de Campaña de Comunicación que al final del día refleje incremento de las matrículas.
La investigación es de tipo cualitativa, se apoya en grupos focales y entrevistas semi-estructuradas; su base conceptual está definida por los enfoques de marketing de servicios y relaciones públicas lo que supone que los factores antes mencionados constituyen el principal elemento para el desarrollo de una propuesta de comunicación creativa que aporte mayor valor a la oferta de la Institución.

La UPS no cuenta hoy en día con un plan de comunicación que guíe sus esfuerzos a un objetivo previamente definido, sin embargo, realiza actividades como charlas a los estudiantes de tercero de bachillerato, visitas a la Institución y de las autoridades a diferentes colegios de la ciudad, cuñas radiales (principalmente previo a las inscripciones), publicaciones en prensa y participación en invitaciones a canales locales de televisión.

En el siglo XXI donde la información está al alcance de todos y la sociedad del conocimiento es eminente, la creatividad para comunicar a los diferentes públicos lo es aún más, las empresas no solo toman en cuenta técnicas publicitarias basadas en medios masivos como principal canal de comunicación, sino incluso otras no tradicionales que permiten también obtener una respuesta inmediata por parte del cliente (Red Gráfica Latinoamérica, 2014).

\section{Bases conceptuales para la estructuración de la metodología}

La conceptualización de la metodología articula técnicamente los enfoques de marketing de servicios y de relaciones públicas, mismos que se exploran a continuación:

Varios autores han discutido acerca del concepto de marketing, algunos como la American 
Marketing Asociation (2013) enfocan a este proceso únicamente como una transacción, otros como Drucker (1986) se acercan un poco más a los clientes y la satisfacción de sus necesidades mientras que Karunakaran (2008), Ramachandra, Chandrashekara \& Shivacumar (2010) y la autora, se apegan a lo expuesto por Kotler \& Keller (2009) quienes consideran que el marketing es "identificar y satisfacer las necesidades de las personas y de la sociedad" lo que permite la construcción de relaciones rentables en un entorno donde la tecnología es imprescindible y la única variable que se mantiene constante es el cambio (Kotler \& Keller, 2009, p. 5).

Para la autora de la presente investigación, los servicios se apegan mucho más al concepto manejado por Stanton, Etzel y Walker (2004) al contrario de Lamb, Hair y McDaniel (2002) quienes alejan su apreciación del fin último, que radica en la satisfacción del cliente y sus necesidades, por tanto, desde la óptica de Kotler, Bloom y Hayes (2004), el marketing de servicios constituye un proceso de intercambio entre consumidores y organizaciones con el objeto de satisfacer las necesidades de los clientes que integra cuatro características, intangibilidad, inseparabilidad, variabilidad y caducidad lo que obliga a diseñar estrategias de marketing interactivo que garantice mejores relaciones con el cliente.

Los servicios deben ser diseñados en base a los gustos y preferencias del cliente, por tanto, deben priorizarse aquellas estrategias que permitan desarrollar vínculos de lealtad y de alto nivel con estos últimos, algunas de las opciones son: Intensificar la relación con los clientes, esto se logra con la venta de paquetes o servicios complementarios, es decir, deleitar a un cliente vendiéndole tantos servicios como le sea posible y fomento de la lealtad mediante recompensas económicas y no económicas (Lovelock \& Wirtz, 2015).

Acerca del tema, Lovelock (1997) hace mención en que cada vez es mayor la preocupación por convertir al servicio en el factor clave de los negocios y en una estrategia para la competitividad. Para las empresas que prestan servicios, es necesario entender los beneficios específicos que ofrece para lograr estructurar una propuesta de valor original. Por otro lado, si se considera la mezcla del marketing, se puede ubicar a una de las variables clave, la comunicación, que de la mano de las relaciones públicas, guarda estrecha relación con la alta dirección de las empresas, forma y fortalece vínculos con los stakeholders o grupos de interés.

Las relaciones públicas han tomado fuerza y son valoradas particularmente, algunos autores proponen incluso su redefinición, hoy los profesionales de este campo, son los encargados de dirigir de manera estratégica los mensajes al público objetivo así como el manejo en situaciones de crisis. La simultaneidad de actividades y habilidades del sujeto que maneja relaciones públicas, hacen que su actuar sea riguroso y creativo a la vez destacando actualmente el uso de medios digitales (Martini, 1998).

La comunicación en general al ser manejada como una de las variables del marketing permite transmitir un mensaje mediante fuentes variadas, como se menciona anteriormente, los canales tradicionales siguen siendo una buena alternativa pero no la mejor al momento de llegar al cliente; la publicidad, relaciones públicas, esfuerzos de marketing directo, promoción de ventas, ventas personales, exhibiciones, representan en gran medida lo realizado por las empresas de servicios, sin embargo, existen alternativas nuevas como los mensajes transmitidos por internet, el aprovechamiento del sitio web de la empresa, la publicidad en línea, los video juegos y activaciones que acarrean menor inversión pero mayor impacto y alcance (Lovelock \& Wirtz, 2015).

El papel de las comunicaciones de marketing, de una u otra forma es esencial para las organizaciones, va mucho más allá de un anuncio publicitario, la atmósfera del local, utilización de colores y elementos gráficos, la apariencia y conducta de los empleados, presencia en internet; son atributos que en conjunto crean una impresión de la marca en los clientes, el objeto es lograr una sincronización que logre persuadir al cliente y deleitarlo siempre, por tanto, depende a la vez de estos mismos factores, la formación de las expectativas y de ellas, la satisfacción del cliente. La oferta de valor que diseñe la empresa debe enmarcarse en una experiencia de marca, es importante hacer promesas que los procesos puedan superar (Lovelock \& Wirtz, 2015). 


\section{Metodología}

La presente investigación es analítica, descriptiva, no experimental, de tipo transversal y enfoque mixto. La población seleccionada para el estudio abarca estudiantes de último año de cinco colegios de Cuenca de los cuales la UPS registra mayor ingreso, estos, vienen desarrollando conjuntamente con la universidad un proyecto de refuerzo para ingreso a la Universidad denominado "Matemáticas para Universitarios", los padres de familia de los mencionados alumnos y docentes de último año de colegio, así también los alumnos de Ingeniería del área de ciencia y tecnología de la UPS (Carreras técnicas con que cuenta la Universidad).

La investigación se sustentó en el uso de dos técnicas de recopilación de datos, tres grupos focales y 15 entrevistas semi-estructuradas. Las variables utilizadas en el estudio se establecen en dos dimensiones, una de ellas corresponde a los estudiantes y la otra a la universidad, la primera deriva dos enfoques, uno socioeconómico que define la estratificación de los segmentos a investigar y posteriormente con la definición del discurso a trasmitir; el segundo enfoque es el motivacional a partir del cual se estructuraron las entrevistas. De acuerdo a la segunda dimensión, se considera tanto la propuesta actual de la UPS como la competencia que a nivel nacional la conforman la Escuela Politécnica Nacional, Escuela Superior Politécnica del Litoral, Escuela Politécnica del Ejército, Escuela Superior Politécnica del Chimborazo, Pontificia Universidad Católica de Quito, Universidad Central del Ecuador, Universidad de Cuenca, Universidad del Azuay, Universidad San Francisco de Quito, Universidad Técnica de Ambato y la Universidad Técnica Particular de Loja entre tanto, en el ámbito local está dada por la Universidad de Cuenca, Universidad del Azuay y Universidad Católica de Cuenca.

\section{Ilustración 1. Dimensiones de las variables}

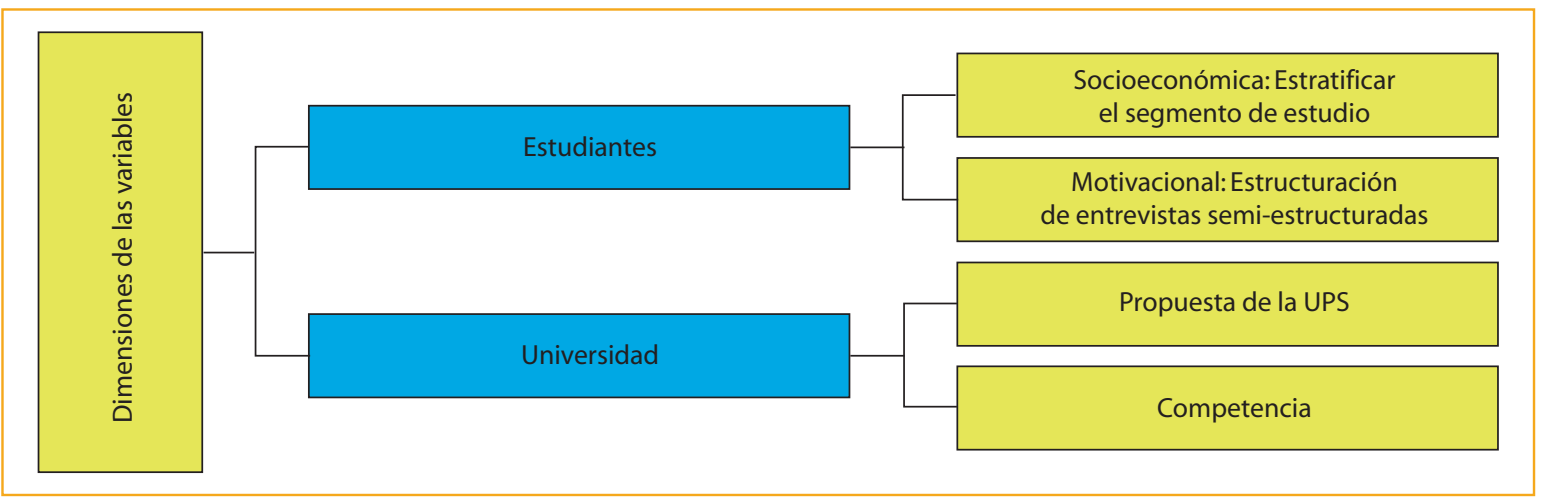

Fuente: Elaboración propia.

En atención a lo expuesto por Malhotra, Dávila y Treviño (2004) y Mejía, Navarrete (2000), se definen los parámetros necesarios para el cálculo de la muestra objeto de estudio; se parte de poblaciones específicas tales como, los alumnos de último año de los colegios de mayor procedencia a la UPS, sus padres, los alumnos de primer año de la Universidad Politécnica Salesiana y los docentes de estos últimos.

\section{Grupos Focales}

Se realizó con estudiantes de cinco colegios de la ciudad de Cuenca, se consideró la variable socioeconómica por la brecha presentada entre las instituciones, encontrándose el primer grupo alineado a un segmento medio alto, el segundo a otro medio bajo y el tercero a un segmento bajo.

En la primera categoría, están ubicados los colegios particulares y fiscomisionales del área 
urbana de Cuenca, en la segunda categoría los Colegios Fiscales del área urbana de Cuenca y en la tercera un Fiscal pero de área rural.

De cada colegio se escogió a tres estudiantes de cursos distintos con la finalidad de disminuir la probabilidad de tener un líder de opinión en el grupo focal.

1. Instituto Técnico Superior Salesiano Colegio Hermano Miguel La Salle

2. Colegio Daniel Córdova - Colegio Manuel J. Calle.

3. Colegio Guillermo Mensi del Valle

\section{Entrevistas semi-estructuradas}

En el caso de las entrevistas, en atención a lo expuesto por Malhotra, Dávila y Treviño (2004) la muestra está dada por la multiplicación de las variables escogidas en cada caso.

- Padres de familia de los Colegios previamente descritos: Para el muestreo se ha considerado las categorías seleccionadas y género.

\section{Cantidad de entrevistas: 6}

- Alumnos de Primer año de la Universidad Politécnica Salesiana Sede Cuenca. (Carreras técnicas) Se considera la cantidad de carreras técnicas que oferta la UPS.

\section{Cantidad de entrevistas: 7}

Docentes de último año de Colegios seleccionados. Se considera como variable el género.

\section{Cantidad de entrevistas: 2}

Los temas a tratarse dentro de las dos técnicas de investigación electas giraron en torno a la decisión de carrera Universitaria, razones de elección, preparación previo a entrada a la Universidad, Instituciones que consideró como opción, conocimiento de cada una de las opciones, medios por los cuales se informó de la oferta académica y de mayor acceso para los estudiantes.

\section{Análisis y resultados}

\section{Resultados grupos focales}

Los resultados han sido estructurados tomando como base el porcentaje de coincidencia en las respuestas:

- Impacto Bajo: Coincidencia del 0\% al 35\%.

- Impacto Medio: Coincidencia del 35\% al $70 \%$

- Impacto Alto: Coincidencia del 70\% al 100\%

Tabla 1. Resultados Grupos Focales

\begin{tabular}{|lll|}
\hline \multicolumn{1}{|c|}{ CRITERIO } & \multicolumn{1}{c|}{ COINCIDENCIA } & IMPACTO \\
\hline Por qué elegir una carrera técnica & Preferencia o afinidad por las matemáticas & Medio \\
\hline Influencia de la familia & Padres o familiares cercanos sugieren una carrera técnica & Alto \\
\hline $\begin{array}{l}\text { Medio de contacto para información } \\
\text { universitaria }\end{array}$ & Llamada telefónica & Medio \\
\hline & Redes Sociales & Alto \\
Objeto de estudiar carrera técnica & Taller Propio & Alto \\
& Ser su propio jefe & Alto \\
\hline & Facilidad de conseguir trabajo & Alto \\
Elección de universidades & Universidad Politécnica Salesiana & Alto \\
& Universidad de Cuenca & Alto \\
\hline $\begin{array}{l}\text { Factores que impulsan la elección de Univer- } \\
\text { sidades: }\end{array}$ & Universidad del Azuay & Medio \\
\hline $\begin{array}{l}\text { Universidad Politécnica Salesiana } \\
\text { Universidad de Cuenca }\end{array}$ & Buenos profesionales, infraestructura, talleres equipados, & Alto \\
Universidad del Azuay & reconocida en el medio. & Alto \\
\hline Tradiciones familiares & Gratuidad, buenos profesionales. & Medio \\
\hline
\end{tabular}

Fuente: Elaboración propia. 
De lo obtenido en los grupos focales, se puede indicar que el grupo de personas que mayor influencia tienen en la elección de una carrera técnica es la familia, lo que muestra coherencia con lo observado en Edelman (2015), existe la percepción de que resulta más fácil conseguir trabajo al prepararse en una carrera técnica lo que no deja de lado su anhelo principal que es el de contar con su propio taller y ser su propio jefe. El medio idóneo para hacerles llegar un mensaje son las redes sociales y en su mayoría prefieren a la UPS como Institución por su reconocimiento en el medio y el equipamiento de sus talleres lo que puede tomarse como elementos esenciales en la formulación del concepto del mensaje, para obtener mayor asertividad en la comunicación para este nicho de mercado.

Los resultados de las entrevistas semiestructuradas a los diferentes públicos muestran una realidad semejante a la de los grupos focales, donde el único diferenciador está en la consideración mayoritaria de la Universidad Estatal por parte de los padres de familia básicamente por los beneficios que ofrece como gratuidad y calidad en la educación, de donde se infiere que la disponibilidad de ingresos es una variable importante a la hora de seleccionar los estudios.

En el caso de los docentes, tienen la percepción de que los amigos tienen gran influencia en la elección de la carrera y Universidad a pesar de que en ocasiones las sugerencias de los docentes son válidas también. La Investigación en su conjunto permite observar que existen criterios de suma importancia que han resultado de coincidencias entre los públicos analizados los mismos que se ubican en la tabla siguiente:

Tabla 2. Conclusiones de la Investigación cualitativa

\begin{tabular}{|lll|}
\hline \multicolumn{1}{|c}{ Criterio } & \multicolumn{1}{c|}{ Coincidencia } & Impacto \\
\hline Influencia de la familia & Padres o familiares cercanos sugieren una carrera técnica & Alto \\
\hline $\begin{array}{l}\text { Medio de contacto para información } \\
\text { universitaria }\end{array}$ & Llamada telefónica & Medio \\
\hline & Redes Sociales & Alto \\
\hline Objeto de estudiar carrera técnica & Taller Propio & Alto \\
& Ser su propio jefe & Alto \\
\hline Tradiciones familiares & Facilidad de conseguir trabajo & Alto \\
\hline
\end{tabular}

Fuente: Elaboración propia.

Al realizar un análisis de los resultados obtenidos, se pueden identificar que las principales motivaciones para elegir una carrera técnica en la Universidad la constituyen el deseo por ser su propio jefe y tener su propio taller o negocio pues la principal percepción identificada es la facilidad en la consecución de trabajo al elegir una carrera técnica, para su desarrollo profesional. Existen casos en que en la familia alguien estudió cierta carrera lo que anima a los jóvenes a continuar con sus estudios al mirar un reflejo suyo a largo plazo, se identifican con profesionales reconocidos, con personas exitosas y los padres por su lado muestran su anhelo de ver a sus hijos como grandes profesionales incluso en algunos casos sin siquiera pensar en el monto de la inversión, si bien todos indicaron que se trata de gustos, puede incidir la experiencia previa que tienen algunos alumnos al encontrarse en un entorno que les permita estar siempre explorando.

Los padres de familia en general tienen personas a su alrededor en las cuales confían y acogen sus consejos, generalmente son amigos y familiares muy cercanos en algunos casos que hayan tenido experiencia con la Institución que sugieren o que hayan escuchado información de la misma (Grupos de interés). 
En coincidencia con Gil, Torres y López (2013) quien en su artículo "Dimensión integral e Interdisciplinaria del comportamiento del consumidor", indica que solo luego de comprender al consumidor se pueden destacar estrategias de comunicación y marketing que realmente lo hagan sentir satisfecho, se indica que el discurso que debe manejar la UPS debe conjugar los resultados expuestos en la tabla 2.

La clave está en desarrollar acciones direccionadas a influenciar de manera directa en los diferentes públicos utilizando como voceros a terceras personas, pues según la información presentada por Edelman (2015) se puede confirmar que las personas que mayor credibilidad tienen hoy en día son los familiares, amigos y expertos académicos, de ahí que se plantea en la campaña la participación de alumnos de primer año de la Universidad, pues al ser de una edad similar existen mayores posibilidades de que formando grupos de amigos, aquellos de último año de colegio, prefieran a la UPS.

\section{Campaña de Comunicación}

En correspondencia con los resultados encontrados, se propone la realización de una campaña de comunicación que tenga como objetivo informar a los estudiantes de último año de colegio acerca de los beneficios de estudiar en la UPS, persuadir a los alumnos de último año de colegio para que prefieran un título de Ingeniería en una Universidad de prestigio y reforzar la percepción de los padres de familia respecto a la UPS con la finalidad de que se considere como primera opción para la educación de sus hijos.

Por tanto, las metas deberían centrarse en incrementar el número de matriculados en las carreras técnicas y mejorar el posicionamiento de la Universidad en el medio.

\section{Determinación de públicos objetivos UPS}

Ilustración 2. Determinación de Públicos UPS

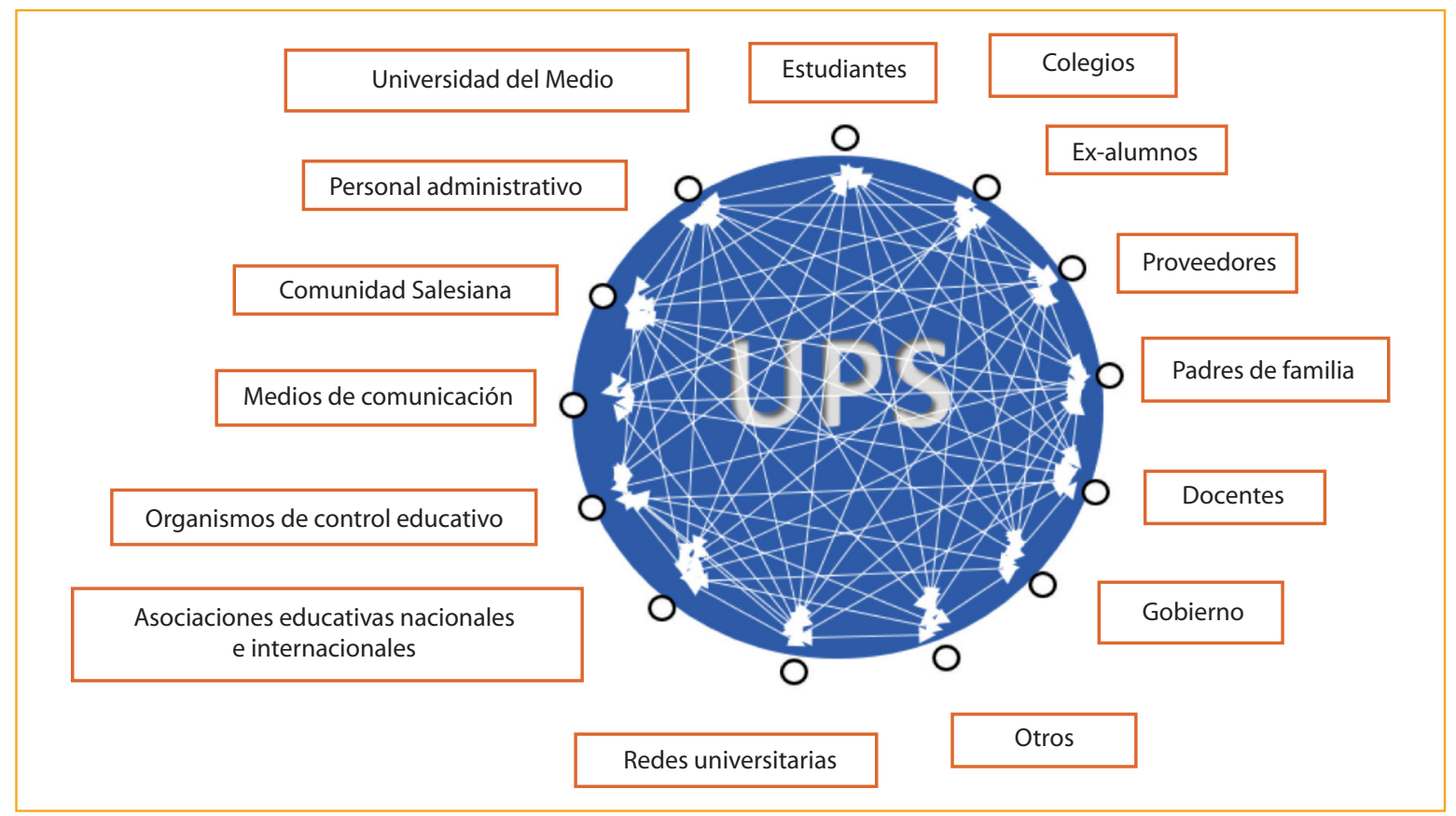

Fuente: Elaboración propia. 


\section{Público Objetivo de la Campaña de Comunicación}

Tabla 3. Público Objetivo de la Campaña de Comunicación

\begin{tabular}{|llll|}
\multicolumn{1}{|c|}{ Públicos } & $\begin{array}{c}\text { Relación de los públicos con } \\
\text { la organización }\end{array}$ & $\begin{array}{c}\text { Resultados esperados } \\
\text { por la organización }\end{array}$ & \multicolumn{1}{c|}{$\begin{array}{c}\text { Expectativa } \\
\text { de los públicos }\end{array}$} \\
\hline $\begin{array}{l}\text { Estudiantes de último año } \\
\text { de colegio. }\end{array}$ & $\begin{array}{l}\text { Potenciales alumnos de la } \\
\text { Universidad. }\end{array}$ & $\begin{array}{l}\text { Elección de la Institución } \\
\text { para educación superior. }\end{array}$ & $\begin{array}{l}\text { Formación de calidad y prefe- } \\
\text { rencia en el mercado laboral. }\end{array}$ \\
\hline $\begin{array}{l}\text { Padres de familia de } \\
\text { alumnos de último año de } \\
\text { colegio. }\end{array}$ & $\begin{array}{l}\text { Financiadores del estudio en } \\
\text { la Institución. }\end{array}$ & $\begin{array}{l}\text { Apoyar y financiar el estu- } \\
\text { dio de sus hijos en la UPS. }\end{array}$ & $\begin{array}{l}\text { Preferencia en el mercado } \\
\text { laboral, desarrollo profesional } \\
\text { de calidad. }\end{array}$ \\
\hline $\begin{array}{l}\text { Docentes de último año de } \\
\text { colegio. }\end{array}$ & $\begin{array}{l}\text { Influenciadores directos en los } \\
\text { alumnos de último año de co- } \\
\text { legio al elegir una universidad } \\
\text { para su preparación. }\end{array}$ & $\begin{array}{l}\text { Influenciar en los alumnos } \\
\text { de último año para que } \\
\text { elijan a la UPS. }\end{array}$ & $\begin{array}{l}\text { Buena preparación de } \\
\text { los jóvenes en el área de } \\
\text { ingenierías. }\end{array}$ \\
\hline
\end{tabular}

Fuente: Elaboración propia.

\section{Acciones propuestas}

En atención a los resultados de investigación obtenidos, las acciones propuestas son:

Tabla 4. Acciones propuestas en la Campaña UPS

\begin{tabular}{|c|c|c|c|}
\hline Acciones & Objetivo & Estrategia & Público \\
\hline $\begin{array}{c}\text { Acción } 1 \\
\text { Implementación de un } \\
\text { CRM. (Customer Relations- } \\
\text { hip Management) }\end{array}$ & $\begin{array}{l}\text {-Implementar un sistema de } \\
\text { CRM. Software que permite } \\
\text { almacenar información de los } \\
\text { estudiantes con la finalidad } \\
\text { de ajustar el servicio a sus } \\
\text { preferencias. }\end{array}$ & $\begin{array}{l}\text { Implementar un software que } \\
\text { almacene adicionalmente a la } \\
\text { información general, datos que } \\
\text { permitan determinar el com- } \\
\text { portamiento de los estudiantes } \\
\text { para desarrollar ofertas acadé- } \\
\text { micas ajustadas a cada perfil. }\end{array}$ & $\begin{array}{l}\text { Potenciales alumnos, } \\
\text { alumnos actuales y ex } \\
\text { alumnos de la UPS. }\end{array}$ \\
\hline $\begin{array}{c}\text { Acción } 2 \\
\text { Desayuno UPS con los } \\
\text { padres de familia de los } \\
\text { alumnos de último año de } \\
\text { colegio. }\end{array}$ & $\begin{array}{l}\text {-Ofrecer información sobre la } \\
\text { oferta de carreras técnicas. } \\
\text {-Analizar la percepción desde } \\
\text { la perspectiva del mercado de } \\
\text { la formación profesional en la } \\
\text { UPS. } \\
\text {-Influenciar en los padres de } \\
\text { familia para que ellos miren a } \\
\text { sus hijos como un reflejo de } \\
\text { los exitosos ex alumnos de la } \\
\text { UPS. }\end{array}$ & $\begin{array}{l}\text { Ofrecer un desayuno a los pa- } \\
\text { dres de familia donde se pue- } \\
\text { da contar experiencias que } \\
\text { motiven a elegir a la UPS por } \\
\text { parte de ex alumnos exitosos } \\
\text { que ocupen altos cargos en } \\
\text { la industria con la finalidad de } \\
\text { que los padres de familia miren } \\
\text { un futuro así para sus hijos al } \\
\text { momento de elegir la UPS. }\end{array}$ & $\begin{array}{l}\text { Padres de familia de los } \\
\text { alumnos de último año de } \\
\text { colegio. }\end{array}$ \\
\hline $\begin{array}{c}\text { Acción } 3 \\
\text { Producción de vídeos } \\
\text { para difundirlos en redes } \\
\text { sociales. }\end{array}$ & $\begin{array}{l}\text {-Influenciar en los alumnos } \\
\text { de último año de colegio para } \\
\text { que al mirar un posible ideal } \\
\text { elijan la UPS. } \\
\text {-Generar orgullo por la UPS } \\
\text { en los alumnos y ex alumnos. }\end{array}$ & $\begin{array}{l}\text { Generar videos donde gran- } \\
\text { des ejecutivos muestren el } \\
\text { valor del título universitario de } \\
\text { la UPS y hablen de las venta- } \\
\text { jas de tener en sus empresas } \\
\text { alumnos graduados de la UPS. }\end{array}$ & $\begin{array}{l}\text { Potenciales alumnos, } \\
\text { alumnos actuales y ex } \\
\text { alumnos de la UPS. }\end{array}$ \\
\hline
\end{tabular}




\begin{tabular}{|c|c|c|c|}
\hline Acciones & Objetivo & Estrategia & Público \\
\hline $\begin{array}{l}\text { Acción } 4 \\
\text { Catálogo digital y físico de } \\
\text { micro currículums de los } \\
\text { graduados de la UPS. }\end{array}$ & $\begin{array}{l}\text {-Incrementar la tasa de gra- } \\
\text { duados con empleo de la } \\
\text { UPS. }\end{array}$ & $\begin{array}{l}\text { Brindar un recurso adicional al } \\
\text { personal de Talento Humano } \\
\text { de varias empresas para con- } \\
\text { siderar a los graduados de la } \\
\text { UPS. }\end{array}$ & $\begin{array}{l}\text { Empresas del Sector. } \\
\text { Alumnos, ex alumnos, }\end{array}$ \\
\hline $\begin{array}{c}\text { Acción } 5 \\
\text { Presencia de marca. }\end{array}$ & $\begin{array}{l}\text {-Incorporar la marca UPS } \\
\text { en los auditorios de la Insti- } \\
\text { tución para el desarrollo de } \\
\text { "publicity". }\end{array}$ & $\begin{array}{l}\text { Incorporar la marca UPS en } \\
\text { los auditorios de la Institución } \\
\text { con el fin de aprovechar la pre- } \\
\text { sencia de marca en medios de } \\
\text { comunicación. }\end{array}$ & $\begin{array}{l}\text { Comunidad en general, } \\
\text { padres de familia. }\end{array}$ \\
\hline $\begin{array}{c}\text { Acción } 6 \\
\text { Experiencia Deportiva } \\
\text { UPS. } \\
\text { Campeonato de futbol } \\
\text { entre los alumnos de último } \\
\text { año de colegios y la UPS. }\end{array}$ & $\begin{array}{l}\text {-Persuadir a los alumnos de } \\
\text { último año de colegio para } \\
\text { que se sientan como en casa, } \\
\text { y conozcan la infraestructura } \\
\text { de la UPS para considerarla } \\
\text { como primera opción }\end{array}$ & $\begin{array}{l}\text { Organizar un campeonato de } \\
\text { futbol entre los estudiantes de } \\
\text { último año de colegio y los pri- } \\
\text { meros ciclos de la UPS con el } \\
\text { objeto de generar una relación } \\
\text { previa entre ellos y por medio } \\
\text { de sugerencias captar mayor } \\
\text { número de estudiantes. }\end{array}$ & $\begin{array}{l}\text { Alumnos de último año de } \\
\text { colegio y de primer año } \\
\text { de Universidad. }\end{array}$ \\
\hline $\begin{array}{c}\text { Acción } 7 \\
\text { Metodología de Enseñanza } \\
\text { - Aprendizaje UPS. }\end{array}$ & $\begin{array}{l}\text {-Desarrollar una metodolo- } \\
\text { gía de enseñanza - apren- } \\
\text { dizaje propia para ganar } \\
\text { reconocimiento de marca y } \\
\text { diferenciación. }\end{array}$ & $\begin{array}{l}\text { Estructurar una metodología } \\
\text { propia por cada carrera de la } \\
\text { institución que conjugue las } \\
\text { mejores prácticas en base a } \\
\text { la evaluación docente y de los } \\
\text { estudiantes. }\end{array}$ & $\begin{array}{l}\text { Alumnos de los últimos } \\
\text { años de colegio. }\end{array}$ \\
\hline $\begin{array}{c}\text { Acción } 8 \\
\text { Influenciadores UPS. }\end{array}$ & $\begin{array}{l}\text {-Influenciar a los docentes de } \\
\text { último año del colegio para } \\
\text { que sugieran a sus alum- } \\
\text { nos a la UPS como principal } \\
\text { opción. }\end{array}$ & $\begin{array}{l}\text { Realizar visitas informativas y } \\
\text { enviar folletería conjuntamente } \\
\text { con artículos con la marca UPS } \\
\text { a los docentes de último año } \\
\text { de colegio. }\end{array}$ & $\begin{array}{l}\text { Docentes de último año } \\
\text { de colegio. }\end{array}$ \\
\hline $\begin{array}{c}\text { Acción } 9 \\
\text { Campus Tech UPS. }\end{array}$ & $\begin{array}{l}\text {-Incentivar el desarrollo tec- } \\
\text { nológico creativo con la fina- } \\
\text { lidad de crear apego a las } \\
\text { carreras técnicas de la UPS. }\end{array}$ & $\begin{array}{l}\text { Organizar un evento tecnoló- } \\
\text { gico - científico en las insta- } \\
\text { laciones de la UPS donde se } \\
\text { premie el mejor proyecto. }\end{array}$ & $\begin{array}{l}\text { Alumnos de último año de } \\
\text { colegio. }\end{array}$ \\
\hline $\begin{array}{l}\text { Acción } 10 \\
\text { Mi Experiencia en } 10 \\
\text { segundos. }\end{array}$ & $\begin{array}{l}\text {-Generar interacción en re- } \\
\text { des sociales con el hashtag } \\
\text { MIEXPERIENCIAUPS. }\end{array}$ & $\begin{array}{l}\text { Invitar a los alumnos a que } \\
\text { cuenten su experiencia de es- } \\
\text { tudiar en la UPS autofilmándo- } \\
\text { se y cargando a las redes. }\end{array}$ & $\begin{array}{l}\text { Alumnos actuales de la } \\
\text { UPS. }\end{array}$ \\
\hline $\begin{array}{c}\text { Acción } 11 \\
\text { Investigación UPS. }\end{array}$ & $\begin{array}{l}\text {-Generar interacción en redes } \\
\text { sociales con los alumnos de } \\
\text { último año de colegio. }\end{array}$ & $\begin{array}{l}\text { General videos cortos produ- } \\
\text { cidos con la carrera de co- } \\
\text { municación social de la UPS } \\
\text { con la finalidad de mostrar } \\
\text { los Laboratorios y los mejores } \\
\text { trabajos realizados en cada } \\
\text { uno de ellos. (ejemplo: Lab. } \\
\text { Automotriz: Vehículo eléctrico, } \\
\text { vehículo de competencia Lab. } \\
\text { Electrónica: robots) Enviar mail } \\
\text { a los alumnos de último año de } \\
\text { colegio. }\end{array}$ & $\begin{array}{l}\text { Alumnos de último año de } \\
\text { colegio }\end{array}$ \\
\hline
\end{tabular}

Fuente: Elaboración propia. 


\section{Discusión y conclusiones}

Los resultados permiten inferir que el mercado reconoce a la marca UPS por sus carreras técnicas lo que limita el conocimiento total de la oferta académica de la institución, por otro lado, el desarrollo continuo de los mismos esfuerzos de comunicación durante varios periodos, ha dado como consecuencia que el público objetivo "se acostumbre" a lo que hay sin despertar su interés de manera creativa. No se evidencia una planificación que atienda a las necesidades de la marca desde una combinación de comunicación y relaciones públicas. Sin duda, la solución al escenario planteado sería el desarrollo de una mezcla de marketing lo que trae consigo el trabajo de variables adicionales que profundizarían el presente trabajo por lo que se proponen como líneas de investigación futuras.

Es importante que la Universidad continúe desarrollando esfuerzos en medios de comunicación masivos, esto fortalece la imagen de la Universidad e informa pero no abarca al público que la investigación planteada determina como "específicos" mismos que deben priorizarse para obtener una mayor efectividad en la comunicación. Es preciso innovar en las vías de comunicación con la intención de generar impacto, captar la atención del potencial estudiante a la vez que se informa.

Es preciso que la empresa genere una oferta de valor en base a la creación de una "experiencia de marca", entregando al cliente la mayor cantidad de servicios posibles de calidad y que le hagan sentir orgulloso de ser parte de la marca. Su diseño sería motivo de análisis posterior pero es claro que el desarrollo de marketing interno puede representar una alternativa clara de apalancamiento.

Por otro lado, en correspondencia con la importancia actual de la matriz productiva $y$ sobre todo el apoyo que brinda el Gobierno en el desarrollo de Institutos Técnicos y tecnológicos, la Universidad Politécnica Salesiana dada su preocupación por continuar formando honrados ciudadanos, buenos cristianos y profesionales de calidad, se ve obligada a proponer acciones que colaboren con la formación de una Ingeniería completa y no se quede únicamente en una tecnología. Las acciones vinculadas a la campaña de comunicación propuesta por la autora pretenden aportar los recursos necesarios para responder a las exigencias de la realidad actual, que trae consigo una mayor presencia de marca, promoción y posicionamiento en el mercado.

En correspondencia a los hallazgos de la investigación, los principales puntos a los que la institución debe prestar atención son:

- Influencia de la Familia: Si bien está claro que quienes deciden son los hijos, también en el caso de existir en la familia personas que han cursado ya determinada carrera influye en gran medida en la elección, de la misma manera la familia participa con sus sugerencias sin que éstas signifiquen una obligación. Los padres de familia y alumnos en su mayoría consideran más fiable la información proporcionada por los amigos, familiares y conocidos, por lo mismo atienden de manera activa su aporte.

- El medio de contacto idóneo para hacerles llegar información a los alumnos de último año de colegio es sin duda el internet, el acceso ilimitado sobre todo en redes sociales, permite que ellos todo el tiempo estén a disposición de la información que se les presente en ese sitio, un buen porcentaje considera que sería pertinente recibir una llamada de la Universidad que proporcione información acerca de la oferta académica.

- En general el público investigado tiene la percepción de una persona que estudia una carrera técnica tiene mayor probabilidad de conseguir trabajo, enlaza la idea de practicidad con facilidad en el mercado laboral. Los alumnos de último año de colegio que se encaminan por una carrera técnica confían en que serán en el futuro su propio jefe y tendrán su propio taller.

- La imagen de la Universidad Politécnica Salesiana permite que el público la valore mucho más por su prestigio, considera que lo que diferencia a la UPS de las otras uni- 
versidades es la infraestructura y el equipamiento de los laboratorios, vincula la calidad de los laboratorios con la de profesionales que forma la Universidad.

- El mejor camino para llegar al público objetivo con el mensaje correcto, es definiendo una propuesta de comunicación creativa que llame la atención de los interesados y los anime a ser parte de la experiencia.

- En base a los resultados obtenidos uno de los esfuerzos que al parecer daría los mejores resultados es la referenciación (estudiantes actuales - estudiantes potenciales) lo que representa un primer paso en prácticas de endomarketing.

\section{Referencias bibliográficas}

American Marketing Association (07 de 2013). American Marketing Association. Recuperado el 11 de 09 de 2015, de American Marketing Association: https://www.ama.org/About AMA/Pages/Definition-of-Marketing.aspx

Banco Central del Ecuador (Enero de 2015). Banco Central del Ecuador. Recuperado el 11 de 02 de 2015, de Estadísticas Macroeconómicas: http://www.bce.fin.ec/index.php/estadisticaseconomicas

Drucker, P. (1986). Management: Tasks, Responsibilities, Practices. New York: Harper Collins.

Edelman (2015). Trust Barometer Edelman 2015. Obtenido de http://www.edelman.com/2015edelman-trust-barometer-2/trust-and-innova tion-edelman-trust-barometer/global-results/

Gil Hernández, C. Y., Torres Estrada, I. C., \& López Torres, Z.A. (2013). Dimensión integral e interdisciplinaria del concepto de comportamiento del consumidor. Anagramas - Universidad de Medellín.

Karunakaran, K. (2008). Marketing Management. Bangalore: Himalaya Publishing House PVT. LTD.
Kotler Philip, Bloom Paul, \& Hayes Thomas (2004). El marketing de Servicios Profesionales. Nueva Jersey: Editorial Paidós SAICF.

Kotler Philip, Keller, Kevin Lane (2009). Dirección de Marketing. México: Pearson Educación.

Lamb, C., Hair, J., \& McDaniel, C. (2002). Marketing (6. ${ }^{\mathrm{a}}$ ed.). México: International Thomson Editores.

Lovelock, C. (1997). Mercadotecnia de un Servicio. México: Prentice Hall Hispanamericana, S.A.

Lovelock, C., \& Wirtz, J. (2015). Marketing de Servicios. México: Pearson.

Malhotra, Naresh, Dávila, J. F., \& Treviño, M. E. (2004). Investigación de Mercados. México: Pearson Educación.

Martini, N. (05 de 1998). RRPP NET - Portal de relaciones públicas. Recuperado el 18 de 02 de 2015, de RRPP NET - Portal de Relaciones Públicas: http://www.rrppnet.com.ar/defrrpp.htm

Mejía Navarrete, J. (2000). El muestreo en la Investigación Cualitativa. Investigaciones Sociales, 4(5). pp. 165-180.

Ramachandra, K., Chandrashekara, B., \& Shivacumar, S. (2010). Marketing Management. New Delhi: Himalaya Publishing House.

Red Gráfica Latinoamérica. (05 de 09 de 2014). Red Gráfica Latinoamérica. Obtenido de Red Gráfica Latinoamérica: http://redgrafica.com/ El-concepto-BTL

Samaniego, P. (2013). Las tendencias son complejas para la industria ecuatoriana. Líderes. Recuperado el 18 de 02 de 2015, de http:// www.revistalideres.ec/lideres/pablo-samaniego-tendencias-son-complejas.html

Secretaría de Educación Superior, C. T. (2014). Secretaría de Educación Superior, Ciencia, Tecnología e Innovación. Recuperado el 08 de 12 de 2014, de Secretaría de Educación Superior, Ciencia, Tecnología e Innovación: http://www.senescyt.gob.ec/Institutos/index. $\mathrm{php} /$ reconversion

Stanton, W., Etzel, M., \& Walker, B. (2004). Fundamentos de Marketing (13. a ed.). México: Mc. Graw Hill. 